\title{
Long non-coding RNA SNHG1 promotes breast cancer progression by regulation of $\mathrm{LMO4}$
}

\author{
XIANG XIONG ${ }^{1}$, YEQIAN FENG $^{2}$, LUN LI $^{3}$, JIA YAO $^{3}$, MEIRONG ZHOU $^{3}$, PIAO ZHAO $^{3}$, \\ FEILONG HUANG ${ }^{3}$, LIYUN ZENG $^{3}$ and LIQIN YUAN ${ }^{3}$ \\ Departments of ${ }^{1}$ Burn and Plastic Surgery, ${ }^{2}$ Oncology, ${ }^{3}$ General Surgery, \\ The Second Xiangya Hospital, Central South University, Changsha, Hunan 410011, P.R. China
}

Received June 22, 2019; Accepted December 17, 2019

DOI: $10.3892 /$ or.2020.7530

\begin{abstract}
Long non-coding RNA (lncRNA) small nucleolar RNA host gene 1 (SNHG1) was reported to be a critical regulator of tumorigenesis and is frequently deregulated in several cancer types. However, the exact mechanism by which SNHG1 contributes to breast cancer progression has not been fully elucidated. The identification of the molecular mechanism of SNHG1 is important for understanding the development of breast cancer and for improving the prognosis of the patients with this disease. In the present study, increased expression levels of SNHG1 were noted in breast cancer tumors following analysis of differentially expressed lncRNAs between 1,063 tumor and 102 normal tissues derived from The Cancer Genome Atlas Breast Invasive Carcinoma (TCGA-BRCA) dataset. This finding was further validated using 50 pairs of normal and tumor tissues that were collected from patients with breast cancer. Notably, SNHG1 expression was significantly correlated with estrogen receptor (ER)/progesterone receptor $(\mathrm{PR})$ negative status $\left(\mathrm{ER}^{-} / \mathrm{PR}^{-}\right)$and advanced clinical stage in breast cancer tissues. Knockdown of SNHG1 led to cell growth arrest, cell cycle redistribution and cell migration inhibition of breast cancer cells. The miRDB database predicted that miR-573 interacts with SNHG1. RT-PCR confirmed the negative regulation of miR-573 levels by SNHG1 in breast cancer cells and the Dual-luciferase reporter assay confirmed their complementary binding. The repression of miR-573 by SNGH1 decreased LIM domain only 4 (LMO4) mRNA and protein expression levels in the breast cancer cell lines tested and induced the expression of cyclin D1 and cyclin E. In vitro experiments indicated that LMO4 overexpression could reverse siSNHG1-induced cell growth arrest, cell cycle redistribution and inhibition of cell migration in breast cancer cells. Moreover, the tumor xenograft model indicated
\end{abstract}

Correspondence to: Professor Liqin Yuan, Department of General Surgery, The Second Xiangya Hospital, Central South University, 139 People's Road, Changsha, Hunan 410011, P.R. China

E-mail: yuanliqin@csu.edu.cn

Key words: SNHG1, breast cancer, LMO4, proliferation that SNHG1 knockdown inhibited MDA-MB-231 growth in vivo and LMO4 overexpression reversed the tumor growth inhibition induced by SNHG1 knockdown. The present study demonstrated that SNHG1 acts as a novel oncogene in breast cancer via the SNHG/miR-573/LMO4 axis and that it could be a promising therapeutic target for patients with breast cancer.

\section{Introduction}

Breast cancer remains the most common cancer type in women worldwide and accounts for more than $25 \%$ of all cancer types and $15 \%$ of all cancer-related deaths in females (1). Breast cancer is classified into estrogen receptor-positive $\left(\mathrm{ER}^{+}\right)$breast cancer, epidermal growth factor receptor-2-positive $\left(\mathrm{Her} 2^{+}\right)$ breast cancer and triple-negative breast cancer (ER $/$ progesterone receptor-negative ( $\left.\mathrm{PR}^{-}\right) / \mathrm{Her} 2-\mathrm{TNBC}^{-}$) according to the expression of ER, PR and Her2 (2). Hormonal therapy and Her2-targeted monoclonal antibodies have significantly improved the clinical outcome of $\mathrm{ER}^{+}$and Her2 ${ }^{+}$breast cancer $(3,4)$. However, the relatively low response rate and the development of resistance during treatment limits their therapeutic efficacy in numerous $\mathrm{ER}^{+}$or Her2 ${ }^{+}$breast cancer patients $(5,6)$. The treatment of TNBC patients depends solely on chemotherapy, which reduces the overall survival rate (7). Therefore, it is urgent to investigate the molecular mechanisms that promote breast cancer progression and develop new treatment strategies for patients with breast cancer.

The successful application of deep sequencing technologies has disproved the notion that large proportion of non-translated DNA is 'junk' (8). Accumulating evidence demonstrates that non-coding RNAs (ncRNAs) are involved in normal cellular processes and that their deregulation is closely associated with disease progression (9). Based on length, ncRNAs can be divided into short ncRNA (miRNA, siRNA and piRNA) and long ncRNA (lncRNA) (10). Aberrant expression of several lncRNAs has been reported in human diseases, including breast cancer $(11,12)$. However, the number of lncRNAs that have been experimentally identified as oncogenes or tumor suppressors in breast cancer is considerably low (13-15). lncRNAs can regulate gene expression by sponging miRNAs, binding to promoters or directly interacting with proteins (16-18). Small nucleolar RNA host gene 1 (SNHG1) is a recently discovered lncRNA with oncogenic potential in various 
cancer types $(19,20)$. An increase in the levels of SNHG1 was found to promote cancer cell proliferation and migration by sponging several miRNAs $(21,22)$. A recent study revealed that the upregulation of SNHG1 promoted breast cancer cell proliferation and invasion (23). The molecular mechanisms of the contribution of SNHG1 to breast cancer development require further investigation.

LIM domain only 4 (LMO4) is a family member of the LIM-only subclass of LIM proteins. Its expression is tightly regulated in mammary gland and aberrant expression of LMO4 leads to differentiation blockade of mammary epithelial cells (24). Overexpression of LMO4 is frequently observed in several cancer types including breast cancer $(25,26)$. It was previously reported that the transcriptional regulation of LMO4 expression is mediated by p53 in breast cancer (27).

In the present study, the results demonstrated that SNHG1 levels were elevated in breast cancer tumor tissues and cell lines. Knockdown of SNHG1 inhibited cell proliferation and cell migration of breast cancer cells and induced cell cycle arrest at the G2/M phase. Additional analysis demonstrated that SNHG1 could sponge miR-573 to increase LMO4 expression in the breast cancer cell lines tested. Overexpression of LMO4 was able to reverse SNHG1 knockdown-induced cell proliferation and cell cycle alteration in breast cancer cells as demonstrated by in vitro assays. In addition, SNHG1 knockdown inhibited MDA-MB-231 tumor growth in vivo, which was reversed by LMO4 overexpression. Moreover, SNHG1 expression exhibited a positive correlation with LMO4 mRNA expression in breast cancer tumor tissues. The present findings revealed an oncogenic role of SNHG1 in breast cancer and suggested that it may promote cell proliferation and cell cycle progression via the miR-573/LMO4 axis.

\section{Materials and methods}

Bioinformatic analysis. Bioinformatic analysis of SNHG1 expression was performed in 1,063 breast cancer cases and 102 normal breast cases using the Human Cancer Metastasis Database (HCMDB, http://hcmdb.i-sanger.com/). The Cancer Genome Atlas Breast Invasive Carcinoma (TCGA-BRCA) dataset was selected. The prediction of the potential binding site between miR-573 and SNHG1 and LMO4 was carried out by miRDB (http://www.mirdb.org/) and miRanda software (http://www.microrna.org). The PROGgeneV2 (http://genomics.jefferson.edu/proggene/index.php) was used to study the association between LMO4 expression and the overall survival of patients with breast cancer based on the GSE42568 dataset (28).

Human tissue samples. Human breast cancer tumor tissues and matched normal breast tissues were collected from 50 patients with breast cancer at The Second Xiangya Hospital of Central South University from June 2014 to July 2017. All tissues were obtained following surgery of primary breast cancer tumors and were immediately frozen in liquid nitrogen for subsequent experiments. Prior to project initiation, written informed consent was provided by all patients enrolled in the present study and the experimental procedures were conducted under the supervision of the Ethics Committee of the Second Xiangya Hospital of the Central South University. The protocol of the experiments was approved by the Ethics Committee of the Second Xiangya Hospital of the Central South University (approval no. 2014S057).

Cell culture. 293 cells, the human breast epithelial cell line $\mathrm{MCF} 10 \mathrm{~A}$, the human $\mathrm{ER}^{+}$breast cancer cell lines MCF7, and T47D, and the human triple-negative breast cancer (TNBC) cell lines (ER//PR/Her2-) MDA-MB-231 and MDA-MB-468 were purchased from the American Type Culture Collection (ATCC). The cell lines were used within 6 months following receipt. MCF10A cells were cultured in Mammary Epithelial Cell Growth Medium (MEGM; Lonza) supplemented with $100 \mathrm{ng} / \mathrm{ml}$ cholera toxin (Sigma-Aldrich; Merck KGaA). 293, MCF7 and T47D cells were cultured in RPMI-1640 medium (Gibco; Thermo Fisher Scientific, Inc.) supplemented with 10\% FBS (HyClone; GE Healthcare). MDA-MB-231 and MDA-MB-468 cells were maintained in DMEM (Gibco; Thermo Fisher Scientific, Inc.) containing 10\% FBS (HyClone; GE Healthcare). All cell lines were cultured in a humidified incubator with $5 \% \mathrm{CO}_{2}$.

Plasmid construction and cell transfection. The full length of the LMO4 open reading frame was amplified from the cDNA of 293 cells and ligated into a pcDNA3.1 plasmid. Plasmid transfection was performed using Lipofectamine 3000 (Invitrogen; Thermo Fisher Scientific, Inc.) according to the manufacturer protocol. SNHG1 siRNA and control siRNA were purchased from GenePharma. The sequence for SNHG1 siRNA was CAGCAGTTGAGGGTTTGCTGTGTAT. The transfection of SNHG1 siRNA or control siRNA sequences was achieved using LipoRNAiMax reagent (Invitrogen; Thermo Fisher Scientific, Inc.) in serum-free medium and sustained for $5 \mathrm{~min}$ until addition into the culture medium. miR-NC mimic, miR-573 mimic, miR-NC inhibitor and miR-573 inhibitor were purchased from Applied Biological Materials (ABM). The transfection of the mimic or inhibitor sequences was carried out using Lipofectamine 3000.

Establishment of stable cell lines. For stable knockdown of SNHG1, lentiviral particles were prepared by co-transfection of pLko.1-SNHG1 shRNA, pMD2G and pCMV-dR8.91 into 293 cells using Lipofectamine 2000. Following $72 \mathrm{~h}$ of culture, the medium containing lentiviral particles was obtained and filtered through a $0.45-\mu \mathrm{m}$ filter (Millipore). The medium containing lentiviral particles was added to the MDA-MB-231 cells in 6-well plates. Following $48 \mathrm{~h}$ of culture, the culture medium was replaced with fresh medium containing $5 \mathrm{mg} / \mathrm{ml}$ puromycin (Solarbio) for $24 \mathrm{~h}$ to select the cells successfully infected with the lentivirus. For establishment of SNHG1-knockdown and LMO4-overexpression models, MDA-MB-231 cells and shSNHG1 MDA-MB-231 cells were transfected with the pcDNA3.1-LMO4 plasmid using Lipofectamine 3000. Following 24 h of cell culture, the cells were screened with $4 \mathrm{mg} / \mathrm{ml} \mathrm{G} 418$ (Sigma-Aldrich) for the following $72 \mathrm{~h}$ of growth.

RNA extraction and real-time RT-PCR. Total RNA from tissues and cells was prepared using TRIzol reagent (Invitrogen; Thermo Fisher Scientific, Inc.). The separation of nuclear and cytoplasmic RNA was accomplished using 
a PARIS kit (Invitrogen; Thermo Fisher Scientific, Inc.) according to the manufacturer's instructions. Gene expression was detected following reverse transcription of RNA into cDNA using PrimeScript ${ }^{\mathrm{TM}}$ RT Reagent Kit (Takara Bio, Inc.). miR-573 expression was performed using a stem-loop specific primer method. Real-time RT-PCR was conducted using the SYBR Premix Ex Taq (Takara Bio, Inc.). The expression levels of genes or miRNAs was analyzed using the $2^{-\Delta \Delta \mathrm{Cq}}$ method (29). GAPDH and U6 were used as internal controls for gene and miRNA expression analysis, respectively. The primer sequences are listed in Table I.

Protein extraction and western blot analysis. Protein lysates were prepared using RIPA lysis buffer (Sigma-Aldrich; Merck KGaA). The antibodies for LMO4 (cat. no. ab131030; dilution 1:2,000) and GAPDH (cat. co. AMAB91153; dilution 1:10,000) detection were purchased from Abcam and Sigma-Aldrich/Merck KGaA, respectively. Cyclin D1 (cat. no. 2978; dilution 1:2,000) and cyclin E1 (cat. no. 4129; dilution 1:2,000) antibodies were obtained from Cell Signaling Technology. Secondary antibodies for mouse (cat. no. SA00001-1; dilution 1:10,000) and rabbit (cat. no. SA00001-2; dilution 1:10,000) were obtained from Proteintech. Briefly, $25 \mu \mathrm{g}$ proteins per lane were separated on an $8 \%$ SDS gel and transferred to a PVDF membrane. Following transfer, the membrane was blocked in 5\% non-fat milk and incubated in the presence of the primary antibodies overnight at $4^{\circ} \mathrm{C}$. On the next day, the membrane was incubated with secondary antibodies for an additional $1 \mathrm{~h}$ at room temperature. The protein bands were developed using ECL substrate (Thermo Fisher Scientific, Inc.). The intensity of the bands was quantified using ImageJ (V. 1.6.0; National Institutes of Health).

Dual luciferase reporter assay. The 3' untranslated region (3'UTR) of the pGL3 construct containing wild-type LMO4 3'UTR (LMO4 3'UTR-WT) and the pGL3 construct containing wild-type SNHG1 (SNHG1 3'UTR-WT) were prepared by PCR of the cDNA derived from 293 cells. Two site mutations were introduced into the putative seed regions of LMO4 3'UTR-WT and SNHG1 3'UTR-WT in order to produce mutant constructs. The dual luciferase assay was performed using cells that were co-transfected with either wild-type or mutant-type luciferase reporter plasmids, pRL-TK plasmid, miR-NC mimic or miR-573 mimic sequences. The transfections were performed using Lipofectamine 3000. The relative luciferase activity was measured at $24 \mathrm{~h}$ following transfection using a Dual-Luciferase reporter assay system (Promega Corp.) according to the manufacturer's protocol.

Cell proliferation assay. The cell proliferative ability was measured using a Cell Counting Kit-8 (CCK-8; Dojindo Laboratories). On the first day, the cells were seeded in each well of a 96-well plate. On the next day, the cells were transfected with the indicated siRNA, miRNA mimic or miRNA inhibitor sequences. The cell viability was subsequently examined at 24-h time points between days 1 to 4 , by addition of $10 \mu \mathrm{l}$ CCK- 8 solution into the culture medium. The solution was incubated for $2 \mathrm{~h}$ and the medium containing CCK-8 was aspirated from the wells and added to another 96-well plate.
Table I. List of primer sequences used for real-time RT-PCR.

\begin{tabular}{ll}
\hline miRNA/gene name & \multicolumn{1}{c}{ Sequences } \\
\hline miR-573-RT & CTCAACTGGTGTCGTGGAGTCGG \\
& CAATTCAGTTGAGCACAGGGC \\
miR-573-forward & GCCGAGCTGAAGTGATGTGT \\
miR-573-reverse & CTCAACTGGTGTCGTGGA \\
U6-RT & AACGCTTCACGAATTTGCGT \\
U6-forward & CTCGCTTCGGCAGCACA \\
U6-reverse & TGGTGTCGTGGAGTCG \\
SNHG1-forward & AGGCTGAAGTTACAGGTC \\
SNHG1-reverse & TTGGCTCCCAGTGTCTTA \\
GAPDH-forward & AAGGTGAAGGTCGGAGTCA \\
GAPDH-reverse & GGAAGATGGTGATGGGATTT \\
\hline
\end{tabular}

SNHG1, small nucleolar RNA host gene 1; GAPDH, glyceraldehyde-3-phosphate dehydrogenase.

The absorbance at $450 \mathrm{~nm}$ of each well was measured to estimate the cell number.

Cell cycle analysis. Transfected cells were harvested and fixed with ice-cold $70 \%$ ethanol at $4^{\circ} \mathrm{C}$ overnight. The cells were stained with propidium iodide (PI; Sigma-Aldrich; Merck $\mathrm{KGaA}$ ) for $30 \mathrm{~min}$ and analyzed using flow cytometry. The percentage of the cells present in each cell cycle phase was counted.

Cell migration assay. The wound healing assay was applied to detect cell migratory activity. The cells were grown in 6-well plates at $90 \%$ confluence and subsequently transfected with siRNA for $24 \mathrm{~h}$. The cell layer was scratched with a $10-\mu \mathrm{l}$ pipette tip at the central area and subsequently washed with PBS. Serum-free DMEM was added. The images of the wound area were captured at the 0 and $24 \mathrm{~h}$ time points following the initial scratch. The closure areas of all wells were quantified using Image-Pro Plus (V. 6.0; Media Cybernetics).

Tumorigenesis in nude mice. Female, 5-week-old nude mice (BALB/c-null) were purchased from the Shanghai Laboratory Animal Center (Chinese Academy of Sciences, China) and bred under SPF conditions. The present study was approved by the Ethics Committee of the Second Xiangya Hospital of the Central South University. The mice were randomly divided into the three following groups (n=3): MDA-MB-231, shSNHG1-MDA-MB-231 and shSNHG1+LMO4 OE MDA-MB-231. The cells from each group were subcutaneously injected into the mammary armpit of the mouse fat pad. The tumor size was measured every five days with a caliper. The tumor volume was estimated according to the following formula: Volume $=0.5 \times$ LengthxWidth ${ }^{2}$. The mice were sacrificed by decapitation at 35 days following cell injection and the tumors were dissected.

Immunohistochemistry. A total of 10 formalin-fixed, paraffin-embedded biopsy breast tumors were available. Immunohistochemistry was performed as described 

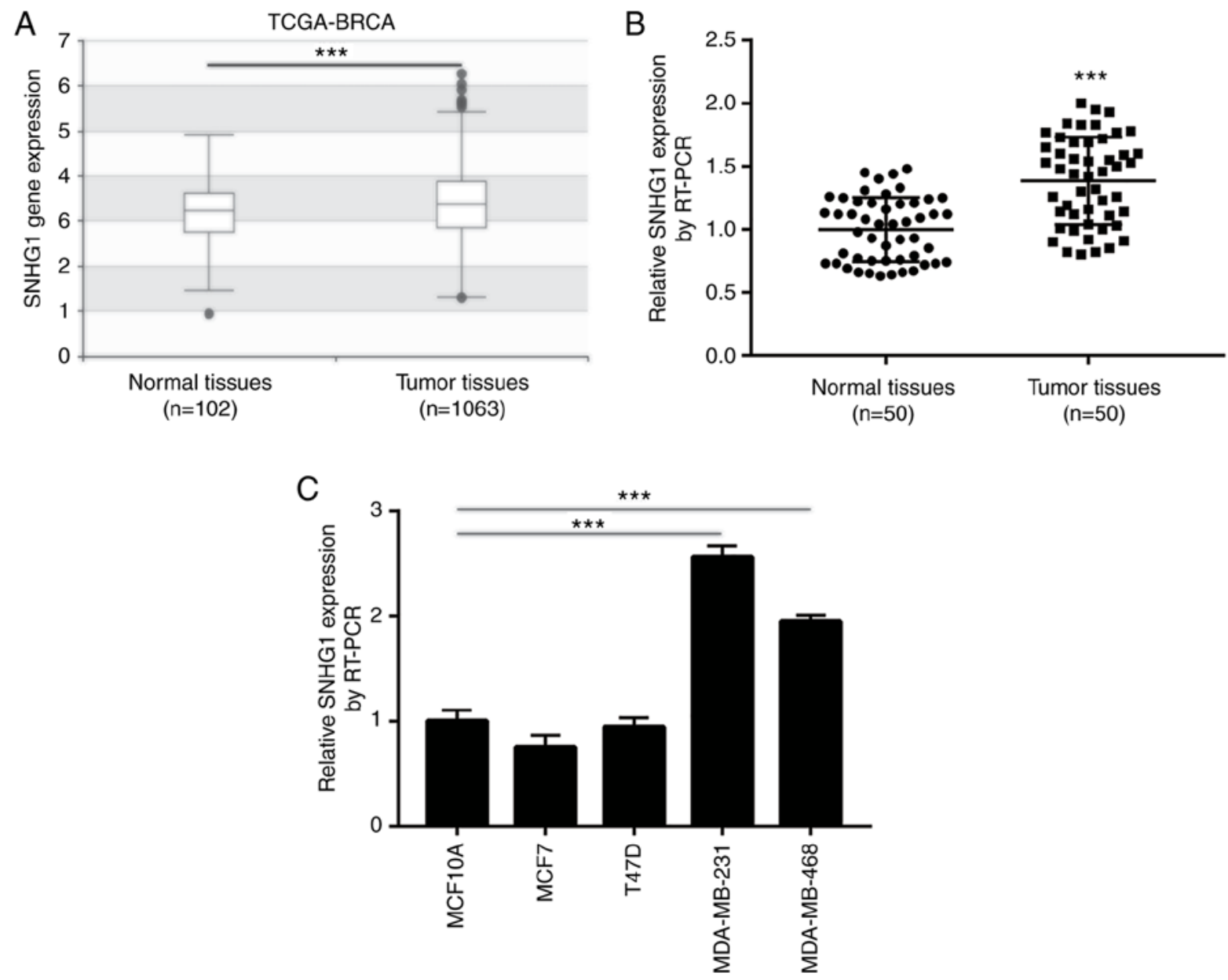

Figure 1. SNHG1 is overexpressed in breast cancer tumor tissues and cell lines. (A) Bioinformatic analysis of the public The Cancer Genome Atlas Breast Invasive Carcinoma (TCGA-BRCA) dataset showed that $S N H G 1$ levels were elevated in 1,063 primary breast cancer tumors compared with 102 normal breast tissues. (B) RT-PCR showed that expression of SNHG1 was increased in 50 breast cancer tumors compared with matched normal breast tissues. (C) Compared with normal epithelial breast cell MCF10A cells, SNHG1 expression was increased in triple-negative breast cancer cell lines MDA-MB-231 and MDA-MB-468. ${ }^{* * *} \mathrm{P}<0.001$. SNHG1, small nucleolar RNA host gene 1 .

previously (30). The samples were stained with the LMO4 antibody (dilution, 1:100) used in the western blotting experiments. Diaminobenzidine (DAB; Boster Inc.) was used for color development. The specimens were observed via a Olympus BX51 light microscope (Olympus Corp.) at x100 magnification.

Statistical analysis. The data presented in the present study were calculated using GraphPad Prism 7 software (GraphPad Software, Inc.) and are represented as mean \pm SD. The comparison between the two groups was achieved using the paired Student's t-test. The differences among the three groups were analyzed using one-way ANOVA followed by the Student-Newman-Keul test. The comparison for more than 3 groups was performed using one-way ANOVA followed by the Tukey's test. The values were considered significantly different at $\mathrm{P}<0.05$.

\section{Results}

SNHG1 is overexpressed in breast cancer tumor tissues and cell lines, notably in ER/PR breast cancer. The expression levels of SNHG1 in breast cancer have not been previously studied. To explore the expression profile of SNHG1 in breast cancer, its expression levels were assessed in 102 normal breast and 1,063 primary breast cancer tissues derived from the TCGA-BRCA dataset. A slight yet significant elevation in SNHG1 levels was observed in breast cancer tissues compared with that noted in normal tissues (Fig. 1A). Subsequently, RT-PCR was applied to detect SNHG1 expression in 50 tissue pairs derived from breast cancer and normal breast tissues. The results demonstrated that SNHG1 expression was significantly higher in breast cancer tissues compared with that noted in normal breast tissues (Fig. 1B). In addition, the increase in the levels of SNHG1 was associated with advanced pathological stage, as well as ER' and PR' status (Table II). However, SNHG1 expression levels were not associated with factors, such as age, lymph node metastasis and Her2 status (Table II). Furthermore, RT-PCR indicated that SNHG1 levels were increased in TNBC MDA-MB-231 and MDA-MB-468 cell lines compared with those noted in the normal epithelial breast cell line MCF10A (Fig. 1C). SNHG1 expression levels were not increased significantly in the $\mathrm{ER}^{+}$breast cancer cell lines MCF7 and T47D compared with those noted in the MCF10A cell line (Fig. 1C). These results indicated that high expression levels of SNHG1 were associated with breast cancer incidence, notably with ER-PR breast cancer. Therefore, MDA-MB-231 and MDA-MB-468 cells were used for the following experiments. 
Table II. Association between clinicopathological variables and SNHG1 expression in 50 breast cancer patients.

\begin{tabular}{|c|c|c|c|c|}
\hline \multirow[b]{2}{*}{ Variables } & \multirow[b]{2}{*}{ No. of cases } & \multicolumn{2}{|c|}{ SNHG1 expression } & \multirow[b]{2}{*}{ P-value } \\
\hline & & High & Low & \\
\hline Age (years) & & & & 0.567 \\
\hline$>50$ & 29 & 13 & 16 & \\
\hline$\leq 50$ & 21 & 12 & 9 & \\
\hline Lymph node metastasis & & & & 0.087 \\
\hline No & 23 & 8 & 15 & \\
\hline Yes & 27 & 17 & 10 & \\
\hline Pathological stage & & & & 0.001 \\
\hline I-II & 18 & 3 & 15 & \\
\hline III-IV & 32 & 22 & 10 & \\
\hline ER status & & & & 0.001 \\
\hline Negative & 20 & 16 & 4 & \\
\hline Positive & 30 & 9 & 21 & \\
\hline PR status & & & & 0.045 \\
\hline Negative & 22 & 15 & 7 & \\
\hline Positive & 28 & 10 & 18 & \\
\hline Her2 status & & & & 0.538 \\
\hline Negative & 35 & 19 & 16 & \\
\hline Positive & 15 & 6 & 9 & \\
\hline
\end{tabular}

SNHG1, small nucleolar RNA host gene 1; ER, estrogen receptor; PR, progesterone receptor; Her2, epidermal growth factor receptor-2. Significant $\mathrm{P}$-values are noted in bold print.

Knockdown of SNHG1 disrupts cell cycle progression and induces cell cycle arrest in breast cancer cells. The increase in the levels of SNHG1 in breast cancer cells suggest a potential oncogenic role of this lncRNA. To evaluate the function of SNHG1 in breast cancer cells, siRNA-mediated knockdown of SNHG1 was performed to assess cell proliferation of MDA-MB-231 and MDA-MB-468 cells. Transfection of the cells with SNHG1 siRNA significantly decreased SNHG1 expression in both MDA-MB-231 and MDA-MB-468 cells compared with control siRNA transfection (Fig. 2A). In MDA-MB-231 cells, knockdown of SNHG1 significantly inhibited cell growth as determined by CCK-8 cell viability assay (Fig. 2B). Although significant activation of cell apoptosis was not observed following SNHG1 downregulation (data not shown), flow cytometric analysis of cell cycle distribution demonstrated that SNHG1 knockdown resulted in cell cycle redistribution with an accumulation of cells in the $\mathrm{S}$ and G2/M phases (Fig. 2C and D). In the MDA-MB-468 cell line, knockdown of SNHG1 also induced cell growth arrest (Fig. 2E). Specifically, the number of MDA-MB-468 cells that accumulated in the G2/M phase was significantly increased (Fig. $2 \mathrm{~F}$ and $\mathrm{G}$ ). These data suggest that SNHG1 may promote breast cancer cell proliferation via the G2/M cell cycle checkpoint.

SNHG1 knockdown inhibits cell migration of breast cancer cells. Tumor metastasis is considered a major mortality cause in breast cancer patients (31). To investigate whether SNHG1 regulates breast cancer cell migration, wound healing assays were performed to detect cell migration following
SNHG1 knockdown. Knockdown of SNHG1 significantly decreased the wound closure area of the MDA-MB-231 cells (Fig. 3A and B), suggesting that SNHG1 regulates the cell migratory activity of breast cancer cells. Similarly, SNHG1 knockdown reduced the cell migratory activity of the MDA-MB-468 cells (Fig. 3C and D).

SNHG1 is located in nuclear and cytoplasmic regions and sponges miR-573 in breast cancer cells. The molecular mechanism of SNHG1 was examined in breast cancer by detecting its nuclear and cytoplasmic localization. Following isolation of the nuclear and cytoplasmic RNA of MDA-MB-231 and MDA-MB-468 cells, RT-PCR analysis demonstrated that nearly half of SNHG1 was present in the cytoplasm (Fig. 4A), suggesting that it may act as an miRNA and ceRNA, as previously reported (32). Bioinformatic analysis using the miRDB software suggested a potential binding site between SNHG1 and miR-573 (Fig. 4B). Based on the prediction, it was speculated that SNHG1 could negatively regulate miR-573 in breast cancer cells. Knockdown of SNHG1 significantly increased miR-573 levels in both MDA-MB-231 and MDA-MB-468 cell lines (Fig. 4C). Moreover, overexpression of miR-573 by transfection of miR-573 mimic significantly decreased SNHG1 expression in both cell lines tested (Fig. 4D). To further validate the direct binding of SNHG1 with miR-573, luciferase plasmids containing wild-type WT and mutant Mut SNHG1 (with two site mutations in the complementary sequence) were constructed. Using dual luciferase reporter assays, co-transfection of miR-573 mimic and WT SNHG1 exhibited 
A
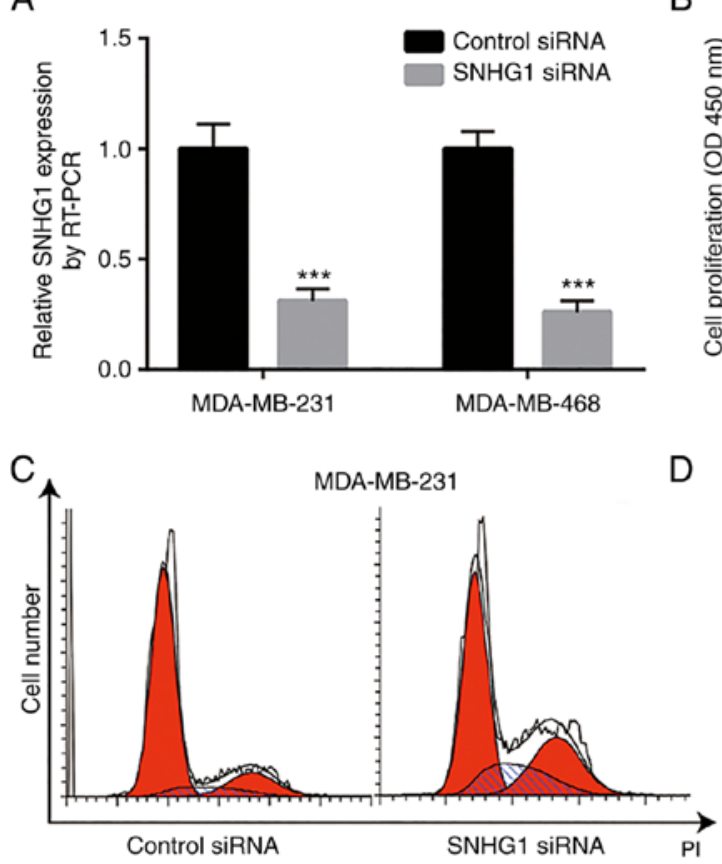

B

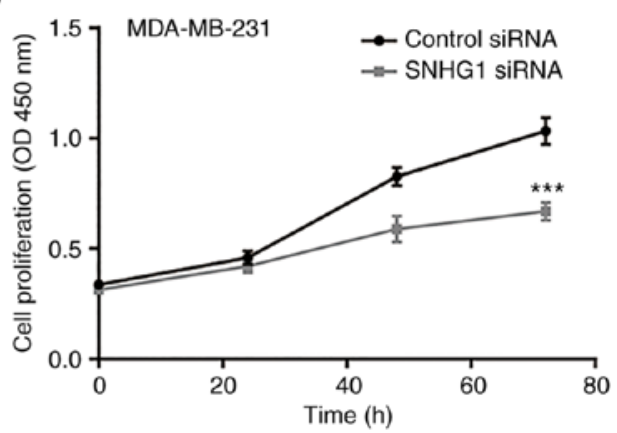

D

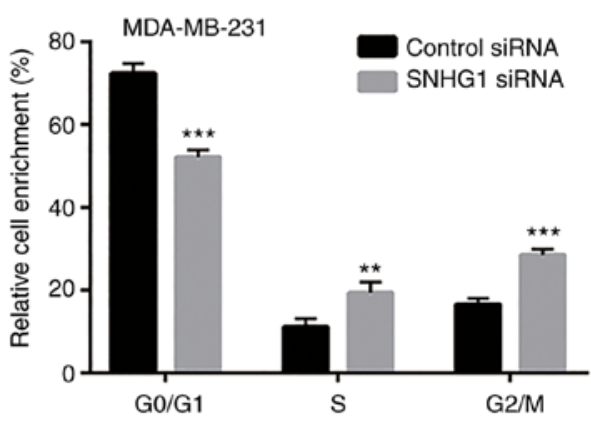

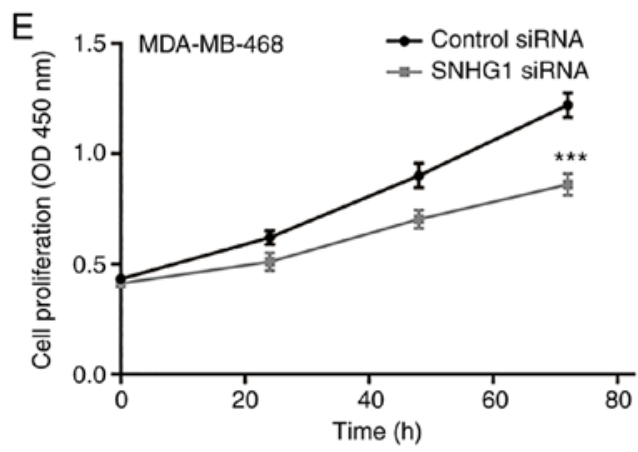
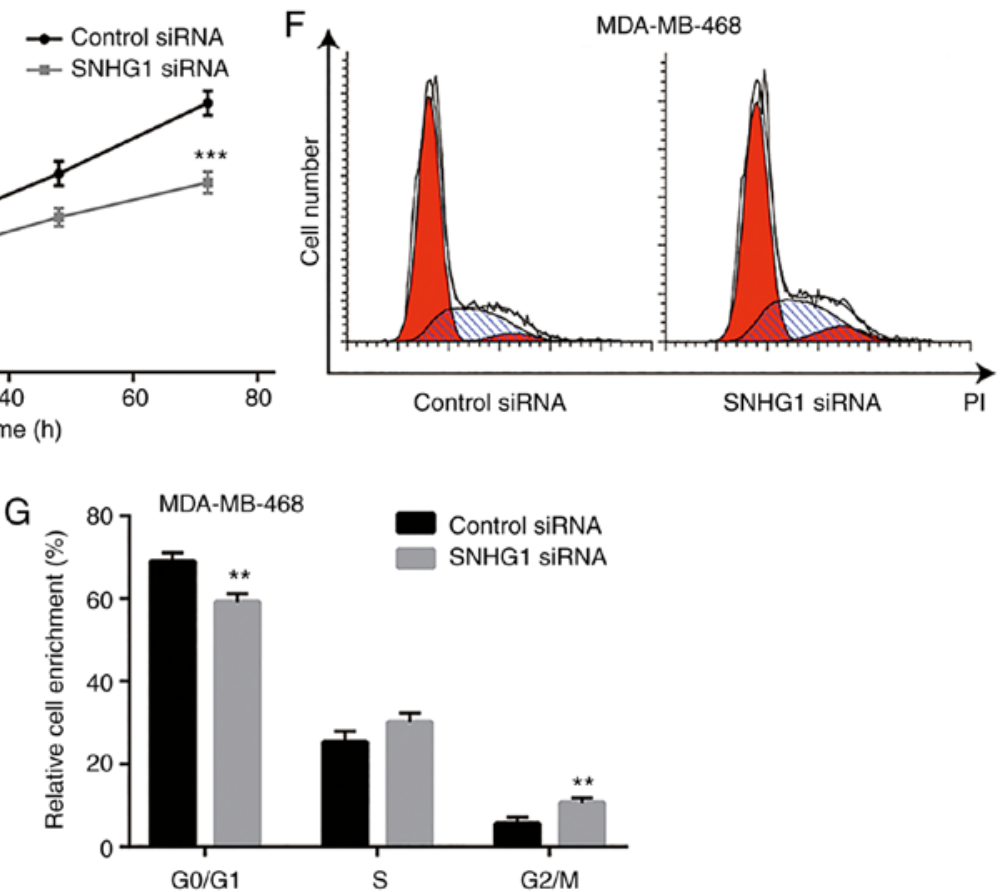

Figure 2. SNHG1 knockdown inhibits the cell proliferation and alters cell cycle progression in breast cancer cells. (A) Transfection of SNHG1 siRNA decreased SNHG1 expression in both the MDA-MB-231 and MDA-MB-468 cells lines. (B) SNHG1 siRNA inhibited the cell proliferation of MDA-MB-231 cells. (C) In MDA-MB-231 cells, SNHG1 knockdown increased the percentage of cells that accumulated in the S and G2/M phases and decreased cells enriched in the G0/G1 phase. (D) Quantification of percentage of cells in the different phases in C. (E) SNHG1 siRNA inhibited cell proliferation of MDA-MB-468 cells. (F) In MDA-MB-468 cells, SNHG1 knockdown increased the percentage of cells that accumulated in the S and G2/M phases and decreased cells enriched in the G0/G1 phase. (G) Quantification of percentage of cells in the different phases in $\mathrm{F} .{ }^{* *} \mathrm{P}<0.01$; ${ }^{* * *} \mathrm{P}<0.001$. SNHG1, small nucleolar RNA host gene 1 .

significantly decreased luciferase activity in the MDA-MB-231 cells (Fig. 4E). Similar results were also observed in the MDA-MB-468 cells (Fig. 4F). Therefore, SNHG1 functions as a negative regulator of miR-573 in breast cancer cells.

miR-573 binds directly to LMO4 to repress its expression. miRNAs regulate gene expression by binding to the 3'UTR of target gene mRNA sequences leading to their degradation or the inhibition of their translation (33). The miRanda software was used to demonstrate that the seed region of miR-573 matched the 3'UTR of LMO4 mRNA (Fig. 5A). LMO4 is an oncogene and is overexpressed in breast cancer (34). Immunohistochemical analysis was used to detect LMO4 expression in 10 breast cancer tumors. Positive expression of LMO4 was observed in the majority of breast cancer tumors (8/10) (Fig. 5B). The prognostic value of LMO4 was analyzed in breast cancer. Based on the GSE42568 $(n=104)$ dataset, high expression levels of LMO4 were associated with reduced overall survival time of the patients 
A
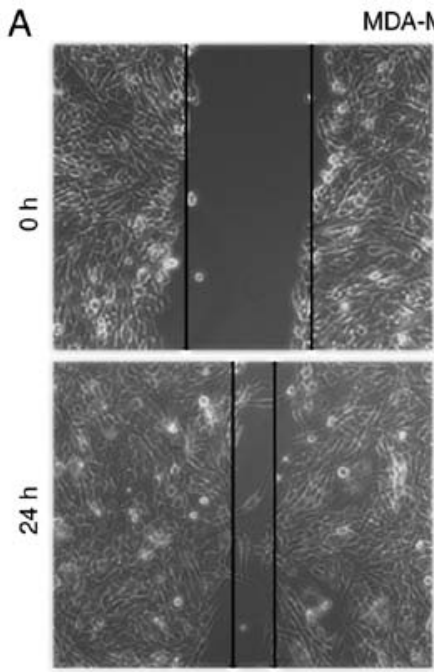

Control siRNA
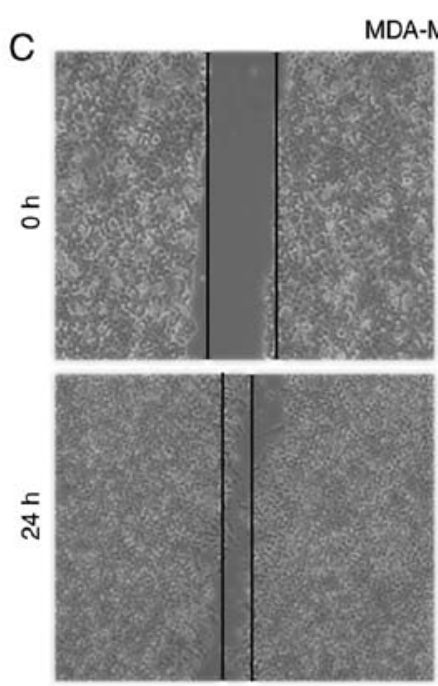

Control siRNA
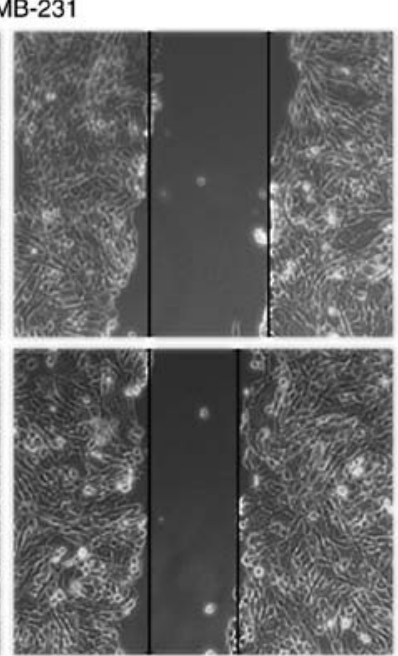

SNHG1 SiRNA
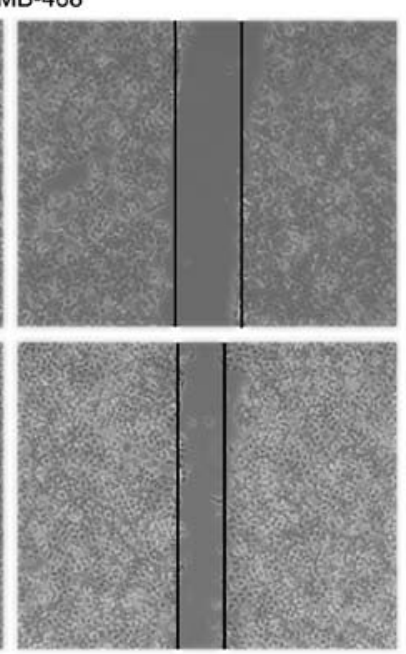

SNHG1 SiRNA
B

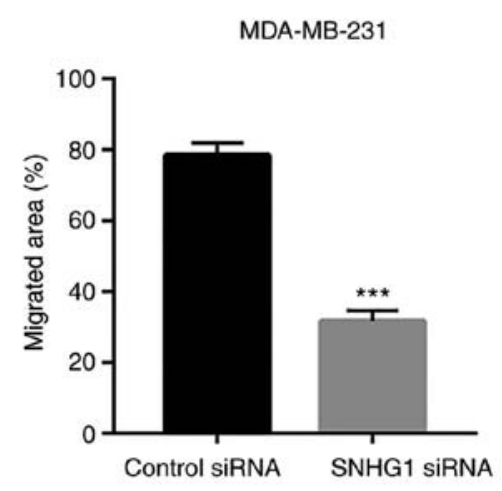

D

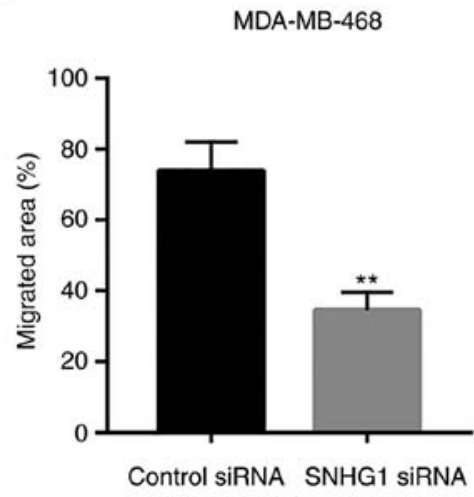

Figure 3. SNHG1 knockdown inhibits cell migration in breast cancer cells. (A) Representative images of the wound healing assay using MDA-MB-231 cells transfected with control siRNA or SNHG1 siRNA. (B) Quantification of the wound closure area in the MDA-MB-231 cells in A. (C) Representative images of wound healing assay using MDA-MB-468 cells transfected with control siRNA or SNHG1 siRNA. (D) Quantification of the wound closure area in the MDA-MB-468 cells in C. ${ }^{* *} \mathrm{P}<0.01 ;{ }^{* * *} \mathrm{P}<0.001$. SNHG1, small nucleolar RNA host gene 1.

with breast cancer (Fig. 5C), suggesting that LMO4 may promote breast cancer progression. In MDA-MB-231 and MDA-MB-468 cells, the transfection of the miR-573 inhibitor significantly increased LMO4 mRNA expression, while the miR-573 mimic decreased LMO4 mRNA levels (Fig. 5D). Western blot analysis further revealed that miR-573 inhibition significantly increased LMO4 protein expression, whereas transfection of the cells with miR-573 mimic significantly reduced LMO4 protein levels (Fig. 5E and F). The direct binding of miR-573 to the LMO4 3'UTR sequence was confirmed in a dual luciferase reporter assay. The overexpression of miR-573 decreased the relative luciferase activity in MDA-MB-231 cells transfected with the LMO4 3'UTR WT sequence, while the luciferase activity of the cells transfected with LMO4 3'UTR Mut sequences was not altered compared with that of the miR-573 mimic (Fig. 5G). Similarly, miR-573 overexpression reduced luciferase activity of MDA-MB-468 cells transfected with the LMO4 3'UTR WT sequence (Fig. 5H). These results indicated that miR-573 directly repressed LMO4 expression in breast cancer cells.
SNHG1 knockdown downregulates LMO4 expression and the expression of key cell cycle regulator proteins in breast cancer cells. The aforementioned results demonstrated that SNHG1 could sponge miR-573, which in turn suppressed LMO4 expression, suggesting that SNHG1 may control LMO4 expression in breast cancer cells. As expected, SNHG1 knockdown led to a decrease in LMO4 mRNA levels in MDA-MB-231 and MDA-MB-468 cells (Fig. 6A). LMO4 is a transcription factor that activates cyclin D1 and E1 transcription and mediates cell cycle progression (35). Knockdown of SNHG1 reduced cyclin D1 and cyclin E1 mRNA levels (Fig. 6A). Moreover, the decrease in the levels of cyclin D1 and cyclin E1 was reversed by transfection of recombinant LMO4 (Fig. 6A). Western blot analysis further indicated that SNHG1 knockdown reduced LMO4, cyclin D1 and cyclin E1 protein levels. These changes were reversed by pcDNA3.1-LMO4 transfection in both cell lines (Fig. 6B and C). The RT-PCR and western blot results revealed the effects of the SNHG1/miR-573/LMO4 axis in breast cancer cell lines. 

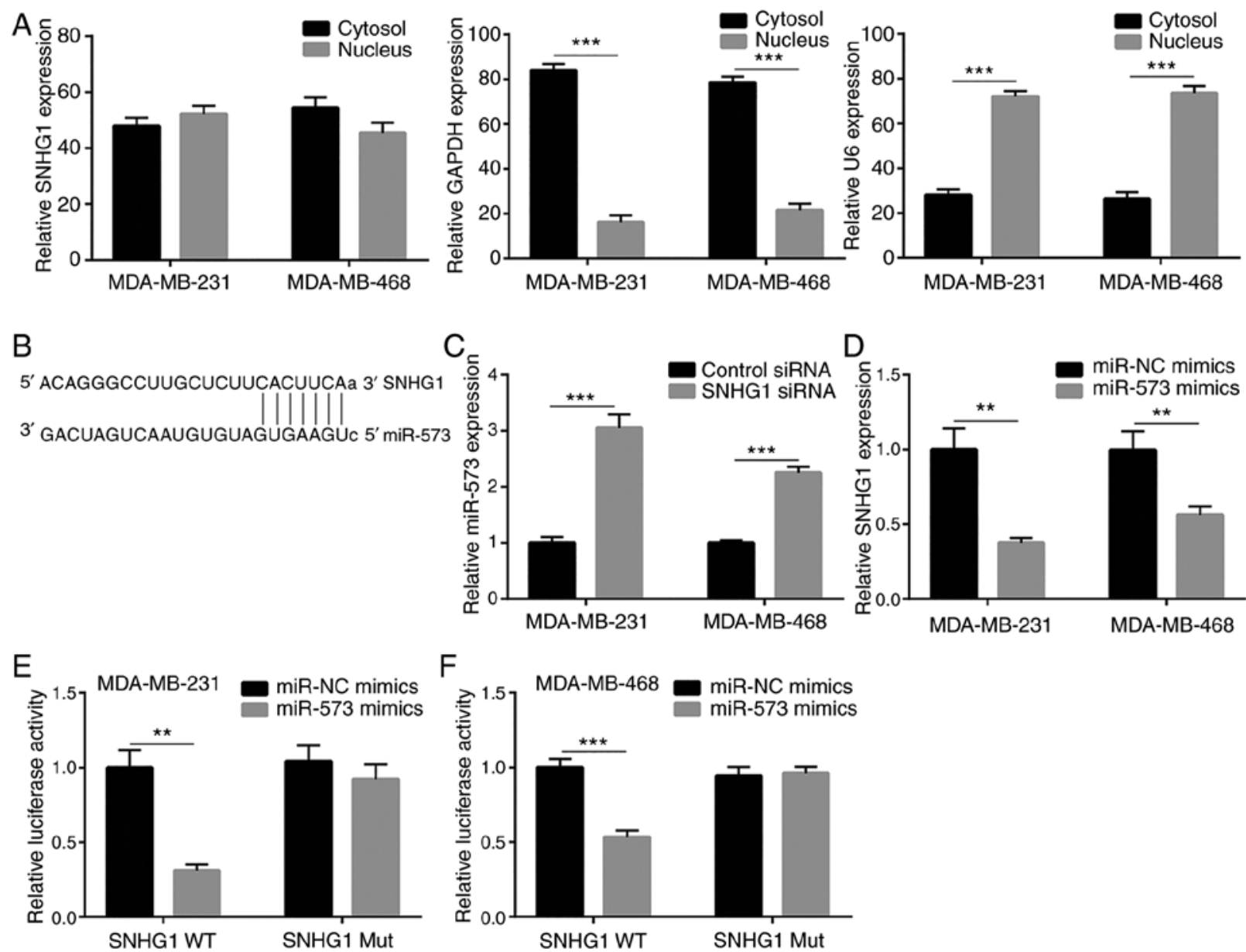

Figure 4. SNHG1 sponges miR-573 to suppress miR-573 levels. (A) The nuclear and the cytoplasmic fractions of SNHG1 RNA from MDA-MB-231 and MDA-MB-468 cells were extracted. SNHG1 expression was measured by RT-PCR. GAPDH was used as a cytosolic marker, and U6 was used as a nuclear marker. (B) Putative matching region between SNHG1 and miR-573 was predicted using miRDB software. (C) SNHG1 knockdown elevated miR-573 levels in MDA-MB-231 and MDA-MB-468 cells. (D) miR-573 mimic decreased SNHG1 levels in MDA-MB-231 and MDA-MB-468 cells. (E) Dual luciferase assay showed that relative luciferase activity was reduced in MDA-MB-231 cells co-transfected with SNHG1 WT and miR-573 mimic. (F) Dual luciferase assay showed that relative luciferase activity was reduced in MDA-MB-468 cells co-transfected with SNHG1 WT and miR-573 mimic. ${ }^{* *} \mathrm{P}<0.01 ;{ }^{* * * *} \mathrm{P}<0.001$. SNHG1, small nucleolar RNA host gene 1; WT, wild-type; Mut, mutant-type.

LMO4 is required for SNHG1-mediated regulation of cell proliferation in breast cancer cells. The regulation of breast cancer cell proliferation by SNHG1 was assessed by cell proliferation of MDA-MB-231 cells transfected with SNHG1 siRNA or SNHG1 siRNA in the presence of pcDNA3.1-LMO4. The increase in the levels of LMO4 partially reversed SNHG1 knockdown-mediated cell growth arrest in MDA-MB-231 cells (Fig. 7A). In addition, overexpression of LMO4 reversed the accumulation of cells at the G2/M phase following SNHG1 knockdown (Fig. 7B and C). Similarly, LMO4 overexpression reversed cell proliferation inhibition and cell cycle redistribution induction by SNHG1 knockdown in MDA-MB-468 cells (Fig. 7D-F). However, overexpression of SNHG1 did not reverse inhibition of cell migration induced by SNHG1 knockdown (data not shown), suggesting that SNHG1 could control cell migration by other mechanisms. These data indicated that LMO4 plays a major role in the SNHG1-mediated cell growth and cell cycle regulation of breast cancer cells.

SNHG1 knockdown inhibits tumor growth in vivo. In addition to the in vitro results, the regulation of breast cancer cell progression by SNHG1 was examined in vivo. MDA-MB-231 cells were infected with a lentiviral vector carrying the SNHG1 or control shRNA sequences to construct stable SNHG1-knockdown and control MDA-MB-231 cell lines. SNHG1 shRNA MDA-MB-231 cells were transfected with pcDNA3.1-LMO4 plasmid and screened with G418 to build LMO4-overexpressing and SNHG1-knockdown MDA-MB-231 cell lines. RT-qPCR indicated that SNHG1 expression was significantly downregulated in the SNHG1 shRNA MDA-MB-231 and SNHG1 shRNA+LMO4 OE MDA-MB-231 cells (Fig. 8A). Western blot analysis confirmed that LMO4 protein expression was decreased in SNHG1 shRNA MDA-MB-231 cells, whereas it was slightly elevated in SNHG1 shRNA+LMO4 OE MDA-MB-231 cells (Fig. 8B and C). Tumor growth was very slow in mice injected with SNHG1 shRNA MDA-MB-231 cells compared with mice in the control and SNHG1 shRNA+LMO4 OE groups (Fig. 8D). Following 35 days of in vivo tumor cell growth, the mice were sacrificed and the tumors were dissected. SNHG1 knockdown significantly reduced the tumor size, whereas overexpression of LMO4 reversed the inhibitory effects on breast cancer cells (Fig. 8E and F). Moreover, the expression levels of SNHG1 and LMO4 were examined in 
A

5' AAAAUCAGUUGAACUUCAAACCUc 3'LMO4 3'UTR $\||||| \mid$

3' GACUAGUCAAUGUGUAGUGAAGUc 5' miR-573
B
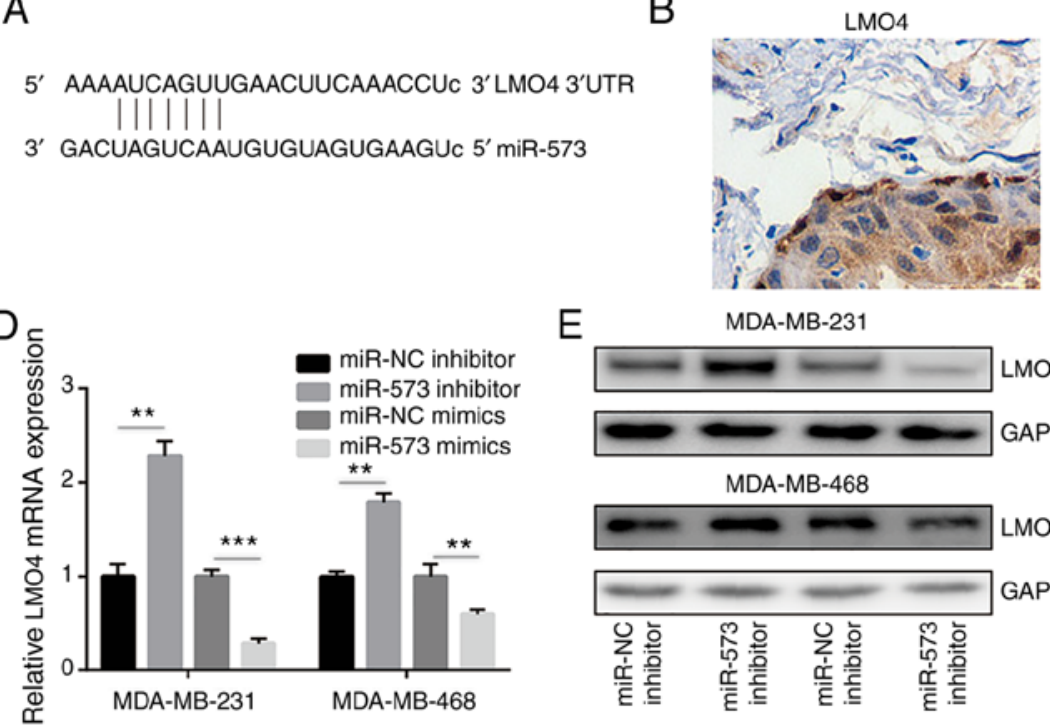

$\mathrm{G} \geqslant 1.5$ MDA-MB-231 $\square$ miR-NC mimics

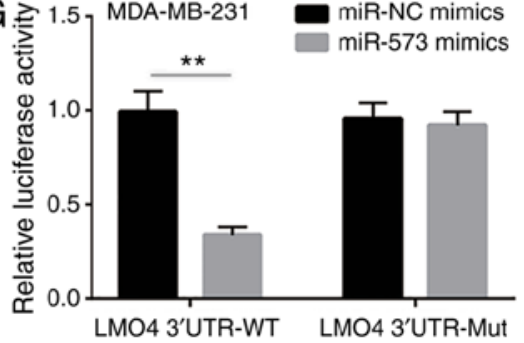

E
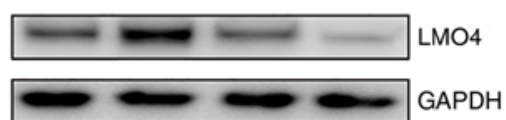

MDA-MB-468
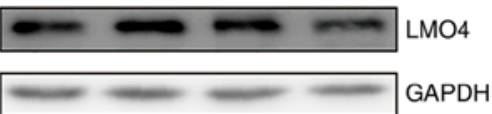

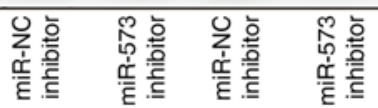

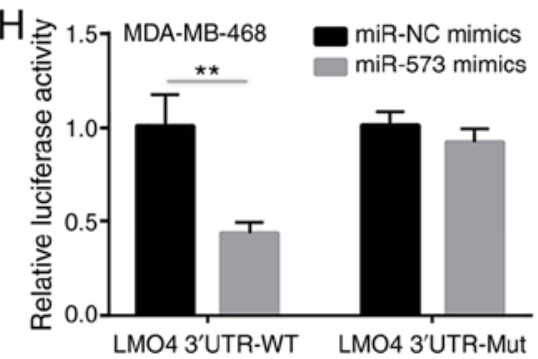

C
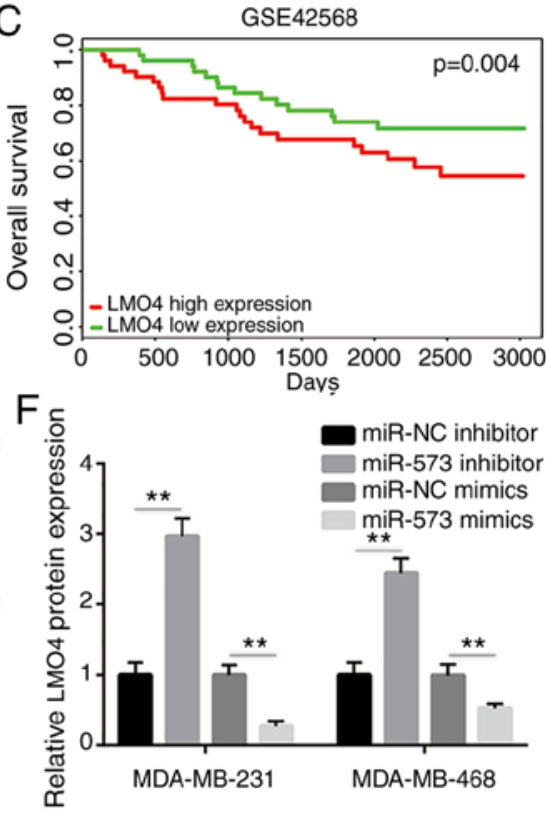

Figure 5. LMO4 is a target gene of miR-573 in breast cancer cells. (A) A putative seed sequence between LMO4 mRNA 3'UTR and miR-573 was predicted using miRanda software. (B) Expression of LMO4 was analyzed by immunohistochemistry (IHC) in 10 breast cancer specimens. Positive LMO4 expression was observed in 9 out of 10 tumors. (C) Association between LMO4 expression and overall survival of patients with breast cancer was analyzed using GSE42958 dataset (n=104). (D) Transfection of miR-573 inhibitor increased LMO4 mRNA levels and miR-573 mimic decreased LMO4 mRNA levels. (E) Transfection of miR-573 inhibitor increased LMO4 protein levels and miR-573 mimic decreased LMO4 protein levels. (F) Quantification of LMO4 protein expression in E. (G) Dual luciferase assay showed that relative luciferase activity was reduced in MDA-MB-231 cells co-transfected with LMO4 3'UTR-WT and miR-573 mimic. (H) Dual luciferase assay showed that relative luciferase activity was reduced in MDA-MB-468 cells co-transfected with LMO4 3'UTR-WT and miR-573 mimic. ${ }^{* *} \mathrm{P}<0.01{ }^{* * *} \mathrm{P}<0.001$. LMO4, LIM domain only 4; WT, wild-type; Mut, mutant-type.
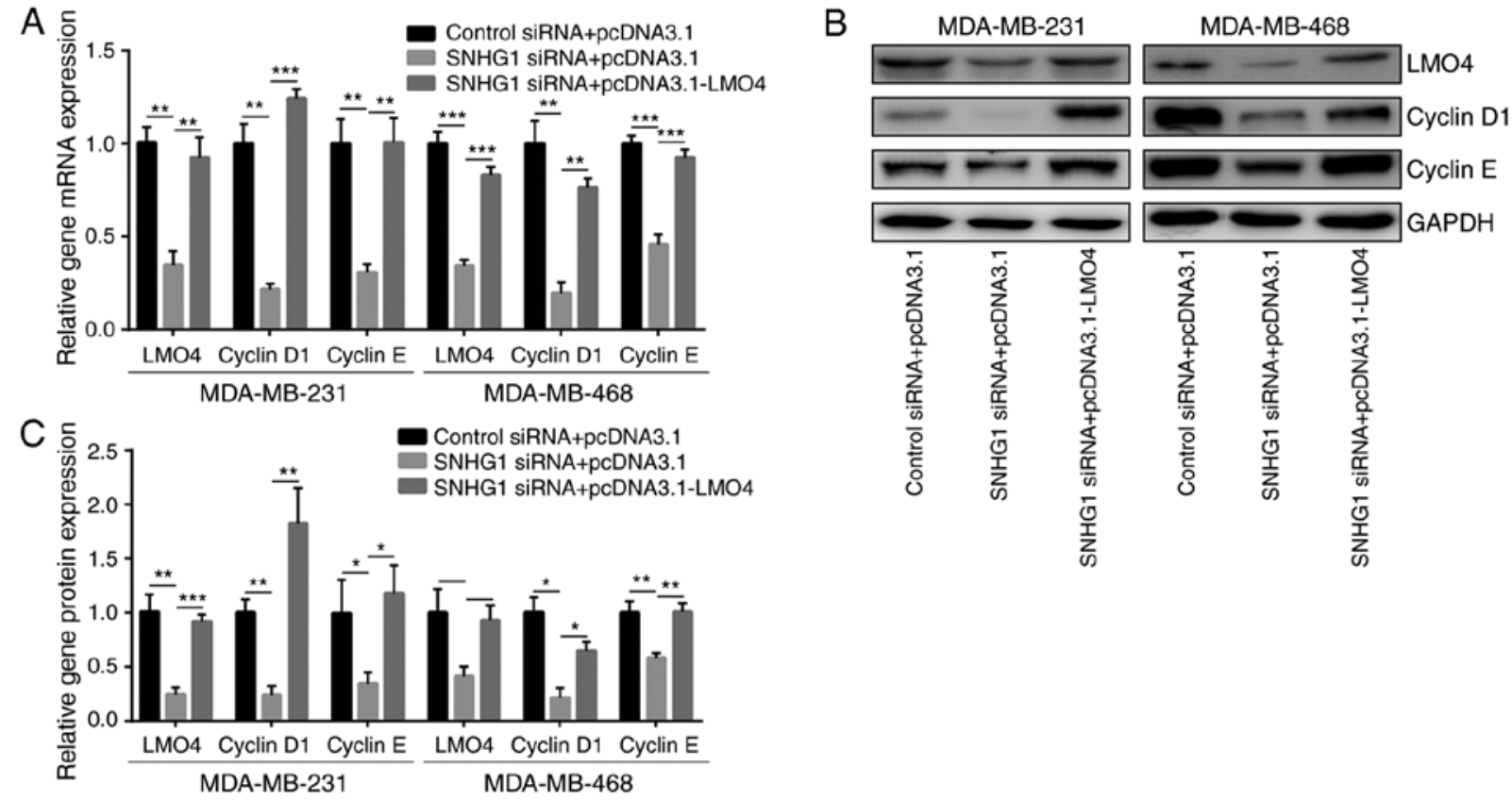

Figure 6. SNHG1 positively regulates $L M O 4$ and its target gene expression. (A) In both MDA-MB-231 and MDA-MB-468 cells, SNHG1 knockdown led to a reduction in LMO4, cyclin D1 and cyclin E1 mRNA levels and was reversed by LMO4 overexpression as determined using RT-PCR. (B) In both MDA-MB-231 and MDA-MB-468 cells, SNHG1 knockdown led to a reduction in LMO4, cyclin D1 and cyclin E1 protein levels and was reversed by LMO4 overexpression using western blot analysis. (C) Quantification of protein expression in $\mathrm{B}$. ${ }^{*} \mathrm{P}<0.05 ;{ }^{* *} \mathrm{P}<0.01 ;{ }^{* * *} \mathrm{P}<0.001$. SNHG1, small nucleolar RNA host gene $1 ;$ LMO4, LIM domain only 4. 


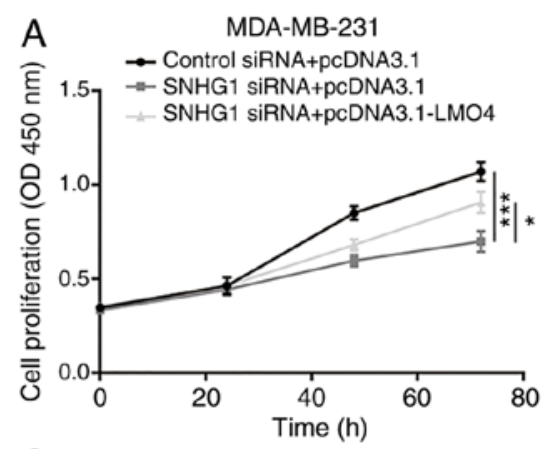

B
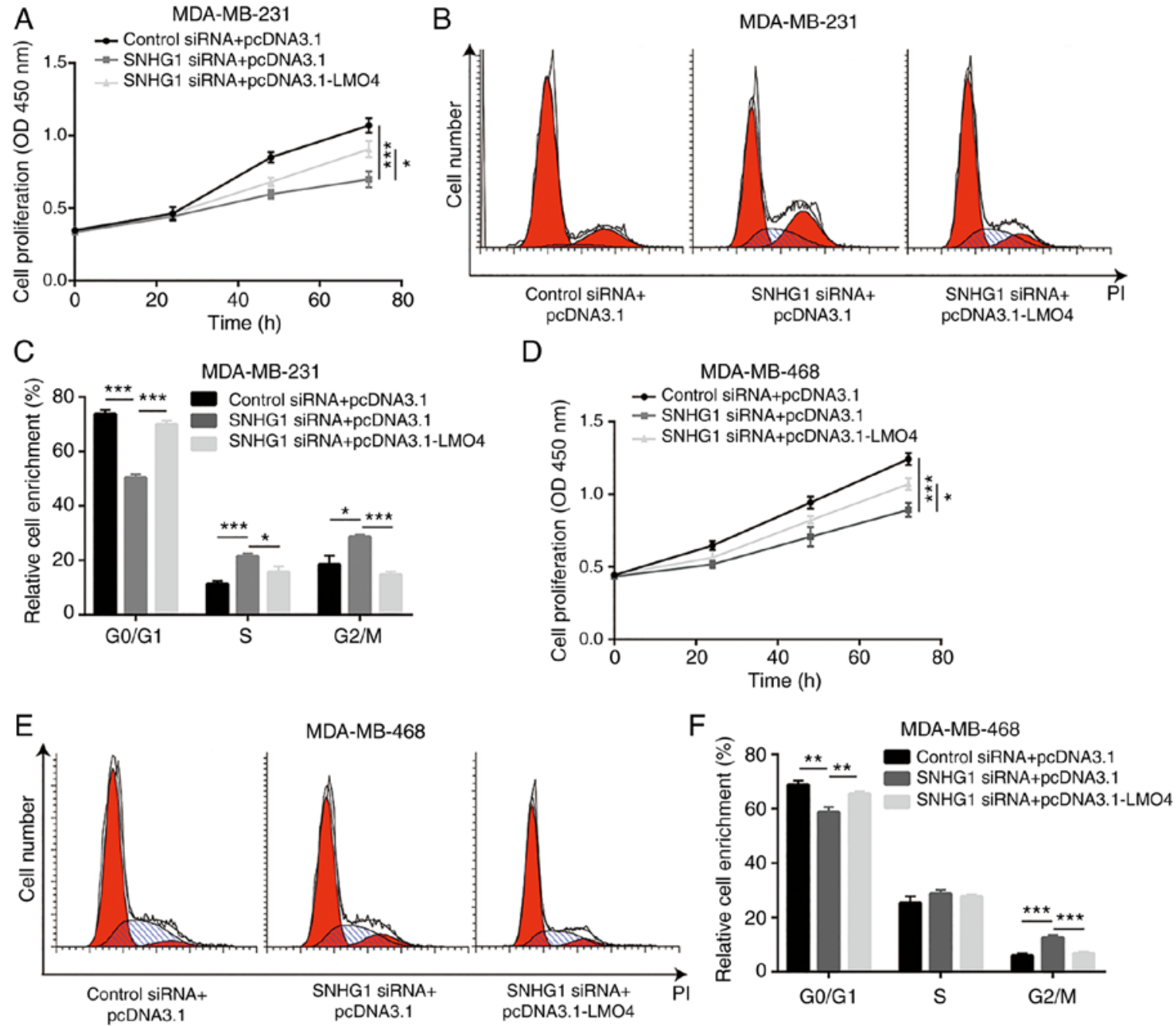

Figure 7. LMO4 is crucial for SNHG1-mediated cell proliferation and cell cycle progression in breast cancer cells. (A) In the MDA-MB-231 cells, LMO4 overexpression reversed the $S N H G 1$ siRNA-induced cell growth arrest. (B) In the MDA-MB-231 cells, LMO4 overexpression reversed the SNHG1 siRNA-induced increase in cell accumulation in the G2/M phase. (C) Quantification of the percentage of cells in the different phases in B. (D) In the MDA-MB-468 cells, LMO4 overexpression reversed SNHG1 siRNA-induced cell growth arrest. (E) In the MDA-MB-468 cells, LMO4 overexpression reversed SNHG1 siRNA-induced increase in cell accumulation in the G2/M phase. (F) Quantification of percentage of cells in the different phases in $\mathrm{E}$. " $\mathrm{P}<0.05$; ${ }^{* *} \mathrm{P}<0.01$; ${ }_{* * * *}^{*}<0.001$. SNHG1, small nucleolar RNA host gene 1; LMO4, LIM domain only 4.

50 breast cancer tumor tissues. The results indicated a positive correlation between SNHG1 expression and LMO4 mRNA expression in tumor tissues (Fig. 8G).

\section{Discussion}

High-throughput deep sequencing and microarray analysis have revealed several differentially expressed long non-coding RNAs (lncRNAs) in breast cancer $(36,37)$. Subsequent experimental analysis identified several IncRNAs which act as oncogenes or tumor suppressors in this type of cancer (38-40). Moreover, the overexpression or downregulation of IncRNAs has been used as a breast cancer biomarker, aiming to improve detection of this disease at an early stage or predict the sensitivity of the patients to chemotherapy (41-43). The present study demonstrated that lncRNA small nucleolar RNA host gene 1 (SNHG1) was overexpressed in breast cancer and that it promoted breast cancer cell growth, cell cycle and migration, suggesting its oncogenic role. These findings are consistent with a recent study (23). The data further revealed a novel molecular mechanism of SNHG1 in breast cancer progression.

Previous studies have shown that high expression levels of SNHG1 predict poor prognosis in hepatocellular carcinoma, glioma, non-small cell lung cancer (NSCLC), colon cancer and prostate cancer (20,44-46). Functional assays indicated that SNHG1 promoted cell proliferation and metastasis of cancer cells, such as glioma and colorectal cancer $(20,45)$. To date, the expression and role of SNHG1 in breast cancer remains elusive. Using the TCGA-BRCA dataset, the present study demonstrated that SNHG1 was significantly elevated in breast cancer tumor tissues compared with the corresponding expression in normal tissues. Subsequently, the overexpression of SNHG1 was confirmed in the tumor counterpart by the detection of SNHG1 expression in 50 pairs of breast cancer and adjacent normal tissues using RT-PCR. Notably, high expression of SNHG1 was associated with $\mathrm{ER}^{-} / \mathrm{PR}^{-}$status and advanced clinical stage. Furthermore, loss of function assays indicated that SNHG1 promoted cell proliferation and cell migration of breast cancer cells. Flow cytometric analysis 
A

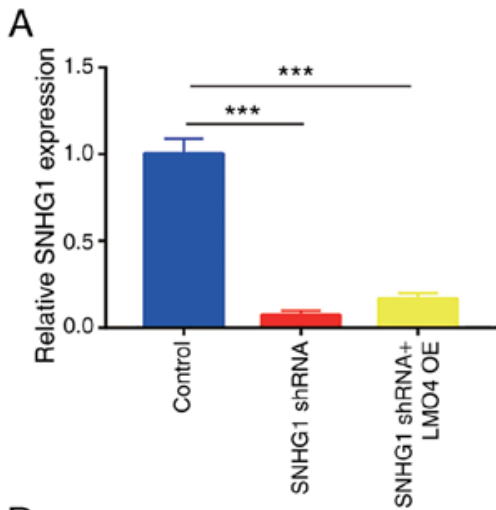

B

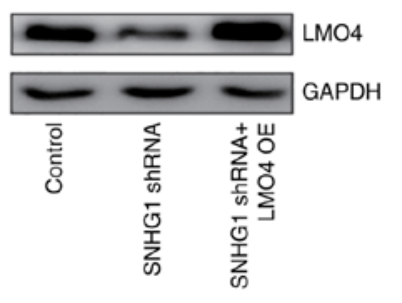

C

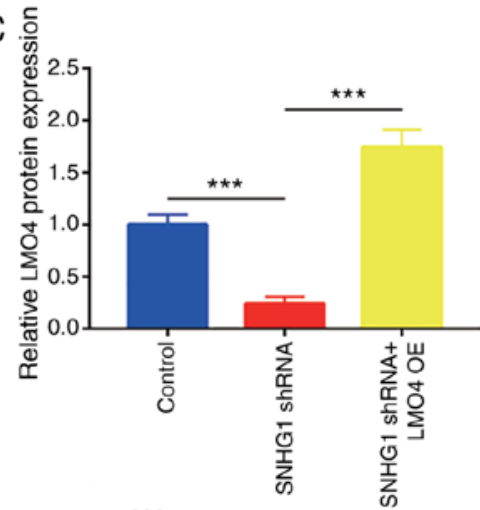

$\mathrm{E}$
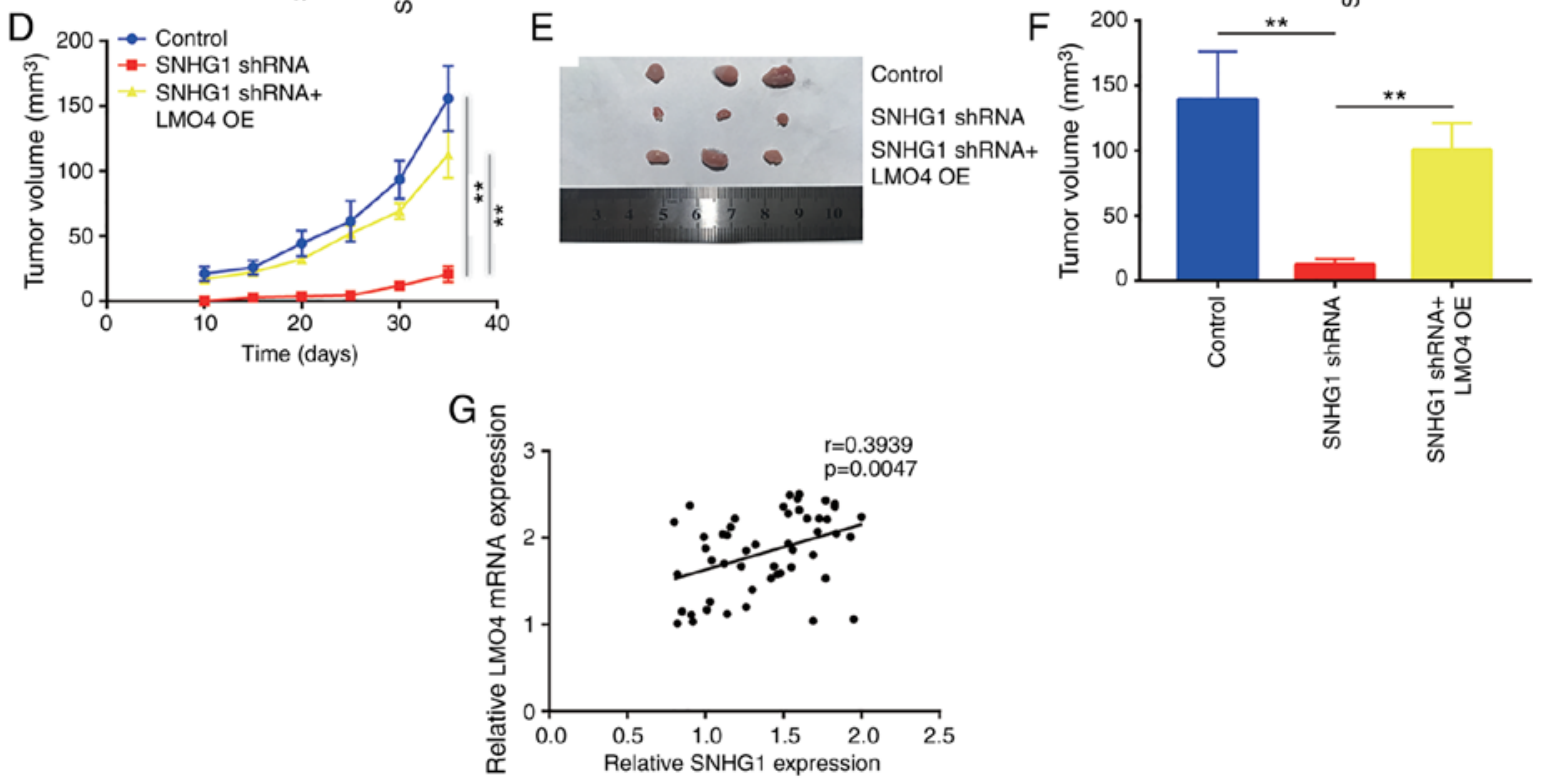

Figure 8. SNHG1 knockdown inhibits tumor growth in vivo. (A) MDA-MB-231 cells were infected with SNHG1 shRNA to construct stable SNHG1-knockdown the MDA-MB-231 cell line. SNHG1 shRNA MDA-MB-231 cells were transfected with the pcDNA3.1-LMO4 plasmid and screened with G418 to construct an LMO4 overexpressing (OE)/SNHG1-knockdown MDA-MB-231 cell line. RT-qPCR showed that SNHG1 expression was decreased in the SNHG1 shRNA MDA-MB-231 and SNHG1 shRNA+LMO4 OE MDA-MB-231 cell lines. (B) Western blot analysis showed that LMO4 protein expression was decreased in the SNHG1 shRNA MDA-MB-231 cells and slightly elevated in the SNHG1 shRNA+LMO4 OE MDA-MB-231 cells. (C) Quantification of LMO4 expression in B. (D) Tumor growth was relatively slow in mice injected with the SNHG1 shRNA MDA-MB-231 cells compared with mice from the control group and SNHG1 shRNA+LMO4 OE group. (E) Tumors were removed from mice after cell injection for 35 days. The tumors were relatively smaller in mice injected with SNHG1 shRNA MDA-MB-231 cells. (F) Quantification of tumor sizes in E. (G) Pearson correlation analysis of SNHG1 and LMO4 mRNA levels showed a positive correlation between their expression in 50 breast cancer tumor tissues. ${ }^{* *} \mathrm{P}<0.01 ;{ }^{* * * *} \mathrm{P}<0.001$. SNHG1, small nucleolar RNA host gene 1; LMO4, LIM domain only 4.

indicated that SNHG1 knockdown led to an accumulation of cells at the $\mathrm{G} 2 / \mathrm{M}$ phase without a concomitant increase in the cell apoptotic rate. These data collectively demonstrated that SNHG1 is overexpressed in breast cancer cells and that it promoted cell proliferation, progression and migration.

The molecular mechanism of SNHG1 is well-characterized in colorectal cancer and NSCLC. Sun et al found that approximately $60 \%$ of SNHG1 is expressed in the nuclei of colorectal cancer and NSCLC cells and that it regulated gene expression of cis and trans elements in order to control AKT signaling activity and MYC levels $(46,47)$. In addition to the regulation of transcription, the cytoplasmic form of SNHG1 functions as a ceRNA in order to determine gene expression. In NSCLC cells, SNHG1 was found to directly bind to miR-145-5p in order to increase MTDH levels (45). In colorectal cancer cells, SNHG1 sponged miR-145 to promote cell proliferation and cell metastasis (22). In the present study, SNHG1 was expressed in both the nuclear and cytoplasmic regions of breast cancer cells. miRDB software was used to predict the potential binding of miRNAs and SNHG1. Among the candidate miRNAs, miR-573 is a well-known tumor suppressor involved in several cancer types $(48,49)$. miR-573 has been reported to target several oncogenes (VEGFA, HIFIA, FAK and ANGPT2) in breast cancer $(48,49)$. Using RT-PCR, a mutual inhibition between miR-573 and SNHG1 was noted in breast cancer cells. Their direct binding was verified using the dual luciferase reporter assay. Furthermore, bioinformatic analysis suggested that the zinc-finger protein $\mathrm{LMO} 4$ is one of the potential target genes of miR-573. LIM domain only 4 (LMO4) expression is frequently elevated in breast cancer (34). Additional experiments in breast cancer cells from various subtypes demonstrated that LMO4 induced cyclin D1 and cyclin E1 expression to promote cell cycle progression and facilitate cell proliferation (35). The expression of LMO4 was regulated by miR-409-3p in colorectal cancer (50). However, its regulation by miRNAs in breast cancer has not yet been investigated. RT-PCR and western blot analyses demonstrated 
that LMO4 is negatively regulated by miR-573. The dual luciferase reporter assay verified that $\mathrm{LMO} 4$ is a target gene of miR-573 in breast cancer cells. Moreover, SNHG1 knockdown decreased LMO4 expression and the expression of its target genes cyclin D1 and cyclin E1. In breast cancer tumor tissues, the expression of LMO4 mRNA was found to be positively associated with SNHG1 levels. The decrease in the expression levels of LMO4 contributed to $\mathrm{G} 2 / \mathrm{M}$ arrest and cell proliferation inhibition in breast cancer cells (35), suggesting that LMO4 may play a major role in SNHG1-induced inhibition of breast cancer progression. These effects were similar to those noted following SNHG1 knockdown. Indeed, the in vitro and in vivo assays presented in the present study revealed that LMO4 overexpression could reverse cell cycle redistribution, cell proliferation inhibition and tumor growth inhibition in breast cancer SNHG1-knockdown mouse xenografts. Zheng et al reported that SNHG1 controlled ZEB1 expression to regulate cell proliferation and migration of breast cancer cells (23). The present findings demonstrated that SNHG1 could modulate cell cycle progression to control cell proliferation in breast cancer cells. The study provided novel insights into the potential mechanism of SNHG1 action in breast cancer progression.

In conclusion, the present study demonstrated that SNHG1 acts as an oncogene in breast cancer. This lncRNA sponged miR-573, leading to elevated levels of LMO4 that in turn promoted cell proliferation and migration. These results support a promising role of SNHG1 in the treatment of patients with breast cancer.

\section{Acknowledgements}

Not applicable.

\section{Funding}

No funding was received.

\section{Availability of data and materials}

The datasets used during the present study are available from the corresponding author upon reasonable request.

\section{Author's contributions}

$\mathrm{XX}, \mathrm{MZ}, \mathrm{PZ}$ and $\mathrm{FH}$ participated in the design and performance of the experiments. XX and JY contributed to the data analysis. YF and LL contributed to the collection of samples and clinical data analyses. LZ and LY supervised the performance of the experiments and data analysis and wrote the manuscript. All authors read and approved the manuscript and agree to be accountable for all aspects of the research in ensuring that the accuracy or integrity of any part of the work are appropriately investigated and resolved.

\section{Ethics approval and consent to participate}

All procedures performed in the present study involving human participants were approved by the Ethic Committee of Second Xiangya Hospital of Central South University (Changsha, Hunan, China). Written informed consent for the publication of any associated data and accompanying images was obtained from all patients prior to surgery.

\section{Patient consent for publication}

Not applicable.

\section{Competing interests}

The authors declare that they have no competing interests.

\section{References}

1. Torre LA, Bray F, Siegel RL, Ferlay J, Lortet-Tieulent J and Jemal A: Global cancer statistics, 2012. CA Cancer J Clin 65: 87-108, 2015.

2. Dent R, Trudeau M, Pritchard KI, Hanna WM, Kahn HK, Sawka CA, Lickley LA, Rawlinson E, Sun P and Narod SA: Triple-negative breast cancer: Clinical features and patterns of recurrence. Clin Cancer Res 13: 4429-4434, 2007.

3. Criscitiello C, Fumagalli D, Saini KS and Loi S: Tamoxifen in early-stage estrogen receptor-positive breast cancer: Overview of clinical use and molecular biomarkers for patient selection. Onco Targets Ther 4: 1-11, 2010.

4. Amiri-Kordestani L, Blumenthal GM, Xu QC, Zhang L, Tang SW, Ha L, Weinberg WC, Chi B, Candau-Chacon R, Hughes P, et al: FDA approval: Ado-trastuzumab emtansine for the treatment of patients with HER2-positive metastatic breast cancer. Clin Cancer Res 20: 4436-4441, 2014.

5. Esteva FJ, Yu D, Hung MC and Hortobagyi GN: Molecular predictors of response to trastuzumab and lapatinib in breast cancer. Nat Rev Clin Oncol 7: 98-107, 2010.

6. Zhu Y, Liu Y, Zhang C, Chu J, Wu Y, Li Y, Liu J, Li Q, Li S, Shi Q, et al: Tamoxifen-resistant breast cancer cells are resistant to DNA-damaging chemotherapy because of upregulated BARD1 and BRCA1. Nat Commun 9: 1595, 2018.

7. Neophytou C, Boutsikos P and Papageorgis P: Molecular mechanisms and emerging therapeutic targets of triple-negative breast cancer metastasis. Front Oncol 8: 31, 2018.

8. Richard JL and Eichhorn PJ: Deciphering the roles of 1ncRNAs in breast development and disease. Oncotarget 9: 20179-20212, 2018.

9. Esteller M: Non-coding RNAs in human disease. Nat Rev Genet 12: 861-874, 2011.

10. Costa FF: Non-coding RNAs, epigenetics and complexity. Gene 410: 9-17, 2008.

11. Ding X, Zhu L, Ji T, Zhang X, Wang F, Gan S, Zhao M and Yang H: Long intergenic non-coding RNAs (LincRNAs) identified by RNA-seq in breast cancer. PLoS One 9: e103270, 2014.

12. Wang J, Ye C, Xiong H, Shen Y, Lu Y, Zhou J and Wang L: Dysregulation of long non-coding RNA in breast cancer: An overview of mechanism and clinical implication. Oncotarget 8: 5508-5522, 2017.

13. Wang M, Wang M, Wang Z, Yu X, Song Y, Wang C, Xu Y, Wei F, Zhao Y and Xu Y: Long non-coding RNA-CTD-2108O9.1 represses breast cancer metastasis by influencing leukemia inhibitory factor receptor. Cancer Sci 109: 1764-1774, 2018.

14. Zheng R, Lin S, Guan L, Yuan H, Liu K, Liu C, Ye W, Liao Y, Jia J and Zhang R: Long non-coding RNA XIST inhibited breast cancer cell growth, migration, and invasion via miR-155/CDX1 axis. Biochem Biophys Res Commun 498: 1002-1008, 2018.

15. Zhao W, Geng D, Li S, Chen Z and Sun M: LncRNA HOTAIR influences cell growth, migration, invasion, and apoptosis via the miR-20a-5p/HMGA2 axis in breast cancer. Cancer Med 7: 842-855, 2018.

16. Tuo YL, Li XM and Luo J: Long noncoding RNA UCA1 modulates breast cancer cell growth and apoptosis through decreasing tumor suppressive miR-143. Eur Rev Med Pharmacol Sci 19: 3403-3411, 2015.

17. Mao C, Wang X, Liu Y, Wang M, Yan B, Jiang Y, Shi Y, Shen Y, Liu $\mathrm{X}$, Lai $\mathrm{W}$, et al: A G3BP1-interacting lncRNA promotes ferroptosis and apoptosis in cancer via nuclear sequestration of p53. Cancer Res 78: 3484-3496, 2018. 
18. Wang H, Li W, Guo R, Sun J, Cui J, Wang G, Hoffman AR and Hu JF: An intragenic long noncoding RNA interacts epigenetically with the RUNX1 promoter and enhancer chromatin DNA in hematopoietic malignancies. Int J Cancer 135: 2783-2794, 2014.

19. Lin SX, Jiang H, Xiang GZ, Zhang WR, Weng YH, Qiu FD, Wu J and Wang HG: Up-regulation of long non-coding RNA SNHG1 contributes to proliferation and metastasis in laryngeal squamous cell carcinoma. Eur Rev Med Pharmacol Sci 22: 1333-1341, 2018

20. Wang Q, Li Q, Zhou P, Deng D, Xue L, Shao N, Peng Y and Zhi F: Upregulation of the long non-coding RNA SNHG1 predicts poor prognosis, promotes cell proliferation and invasion, and reduces apoptosis in glioma. Biomed Pharmacother 91: 906-911, 2017.

21. Tan H, Zhao L, Song R, Liu Y and Wang L: The long noncoding RNA SNHG1 promotes nucleus pulposus cell proliferation through regulating miR-326 and CCND1. Am J Physiol Cell Physiol 315: C21-C27, 2018.

22. Tian T, Qiu R and Qiu X: SNHG1 promotes cell proliferation by acting as a sponge of miR-145 in colorectal cancer. Oncotarget 9 : 2128-2139, 2017

23. Zheng S, Li M, Miao K and Xu H: SNHG1 contributes to proliferation and invasion by regulating miR-382 in breast cancer Cancer Manag Res 11: 5589-5598, 2019.

24. Visvader JE, Venter D, Hahm K, Santamaria M, Sum EY, O'Reilly L, White D, Williams R, Armes J and Lindeman GJ: The LIM domain gene LMO4 inhibits differentiation of mammary epithelial cells in vitro and is overexpressed in breast cancer Proc Natl Acad Sci USA 98: 14452-14457, 2001.

25. Wittlin S, Sum EY, Jonas NK, Lindeman GJ and Visvader JE: Two promoters within the human LMO4 gene contribute to its overexpression in breast cancer cells. Genomics 82: 280-287, 2003.

26. Taniwaki M, Daigo Y, Ishikawa N, Takano A, Tsunoda T, Yasui W, Inai K, Kohno N and Nakamura Y: Gene expression profiles of small-cell lung cancers: Molecular signatures of lung cancer. Int J Oncol 29: 567-575, 2006.

27. Zhou X, Sang M, Liu W, Gao W, Xing E, Lü W, Xu Y, Fan X, Jing S and Shan B: LMO4 inhibits p53-mediated proliferative inhibition of breast cancer cells through interacting p53. Life Sci 91: 358-363, 2012

28. Clarke C, Madden SF, Doolan P, Aherne ST, Joyce H, O'Driscoll L, Gallagher WM, Hennessy BT, Moriarty M, Crown J, et al: Correlating transcriptional networks to breast cancer survival: A large-scale coexpression analysis. Carcinogenesis 34: 2300-2308, 2013.

29. Livak KJ and Schmittgen TD: Analysis of relative gene expression data using real-time quantitative PCR and the 2(-Delta Delta C(T)) method. Methods 25: 402-408, 2001.

30. Xu X, Jin H, Liu Y, Liu L, Wu Q, Guo Y, Yu L, Liu Z, Zhang T, Zhang $\mathrm{X}$, et al: The expression patterns and correlations of claudin-6, methy-CpG binding protein 2, DNA methyltransferase 1 , histone deacetylase 1 , acetyl-histone $\mathrm{H} 3$ and acetyl-histone $\mathrm{H} 4$ and their clinicopathological significance in breast invasive ductal carcinomas. Diagn Pathol 7: 33, 2012.

31. Koleva-Kolarova RG, Greuter MJ, Feenstra TL, Vermeulen KM, de Vries EF, Parkin D, Buskens E and de Bock GH: Molecular imaging with positron emission tomography and computed tomography (PET/CT) for selecting first-line targeted treatment in metastatic breast cancer: A cost-effectiveness study. Oncotarget 9: 19836-19846, 2018.

32. Lan X and Liu X: LncRNA SNHG1 functions as a ceRNA to antagonize the effect of miR-145a-5p on the down-regulation of NUAK1 in nasopharyngeal carcinoma cell. J Cell Mol Med 23: 2351-2361, 2019.

33. Bartel DP: MicroRNAs: Genomics, biogenesis, mechanism, and function. Cell 116: 281-297, 2004.
34. Sum EY, Segara D, Duscio B, Bath ML, Field AS, Sutherland RL, Lindeman GJ and Visvader JE: Overexpression of LMO4 induces mammary hyperplasia, promotes cell invasion, and is a predictor of poor outcome in breast cancer. Proc Natl Acad Sci USA 102: 7659-7664, 2005.

35. Montañez-Wiscovich ME, Shelton MD, Seachrist DD, Lozada KL, Johnson E, Miedler JD, Abdul-Karim FW, Visvader JE and Keri RA: Aberrant expression of LMO4 induces centrosome amplification and mitotic spindle abnormalities in breast cancer cells. J Pathol 222: 271-281, 2010.

36. Wang L, Shen X, Xie B, Ma Z, Chen X and Cao F: Transcriptional profiling of differentially expressed long non-coding RNAs in breast cancer. Genom Data 6: 214-216, 2015.

37. Chen C, Li Z, Yang Y, Xiang T, Song W and Liu S: Microarray expression profiling of dysregulated long non-coding RNAs in triple-negative breast cancer. Cancer Biol Ther 16: 856-865, 2015.

38. Li Q, Gao H, Zhou S and Liao Y: LncRNA PlncRNA-1 overexpression inhibits the growth of breast cancer by upregulating TGF- $\beta 1$ and downregulating PHGDH. Breast Cancer 25: 619-625, 2018.

39. Pan Y, Pan Y, Cheng Y, Yang F, Yao Z and Wang O: Knockdown of lncRNA MAPT-AS1 inhibites proliferation and migration and sensitizes cancer cells to paclitaxel by regulating MAPT expression in ER-negative breast cancers. Cell Biosci 8: 7, 2018.

40. Liu Y, Sharma S and Watabe K: Roles of lncRNA in breast cancer. Front Biosci (Schol Ed) 7: 94-108, 2015.

41. Li J, Wang W, Xia P, Wan L, Zhang L, Yu L, Wang L, Chen X, $\mathrm{Xiao} \mathrm{Y}$ and $\mathrm{Xu} \mathrm{C}$ : Identification of a five-IncRNA signature for predicting the risk of tumor recurrence in patients with breast cancer. Int J Cancer 143: 2150-2160, 2018.

42. Zhang S, Wang J, Ghoshal T, Wilkins D, Mo YY, Chen Y and Zhou Y: IncRNA gene signatures for prediction of breast cancer intrinsic subtypes and prognosis. Genes (Basel) 9, 2018

43. Zhu QN, Wang G, Guo Y, Peng Y, Zhang R, Deng JL, Li ZX and Zhu YS: LncRNA H19 is a major mediator of doxorubicin chemoresistance in breast cancer cells through a cullin4A-MDR 1 pathway. Oncotarget 8: 91990-92003, 2017.

44. Zhang M, Wang W, Li T, Yu X, Zhu Y, Ding F, Li D and Yang T: Long noncoding RNA SNHG1 predicts a poor prognosis and promotes hepatocellular carcinoma tumorigenesis. Biomed Pharmacother 80: 73-79, 2016.

45. Lu Q, Shan S, Li Y, Zhu D, Jin W and Ren T: Long noncoding RNA SNHG1 promotes non-small cell lung cancer progression by up-regulating MTDH via sponging miR-145-5p. FASEB J 32: 3957-3967, 2018.

46. Sun X, Wang Z and Yuan W: Down-regulated long non-coding RNA SNHG1 inhibits tumor genesis of colorectal carcinoma. Cancer Biomark 20: 67-73, 2017.

47. Sun Y, Wei G, Luo H, Wu W, Skogerbø G, Luo J and Chen R: The long noncoding RNA SNHG1 promotes tumor growth through regulating transcription of both local and distal genes. Oncogene 36: 6774-6783, 2017.

48. Wang L, Song G, Tan W, Qi M, Zhang L, Chan J, Yu J, Han J and Han B: MiR-573 inhibits prostate cancer metastasis by regulating epithelial-mesenchymal transition. Oncotarget 6: 35978-35990, 2015.

49. Danza K, De Summa S, Pinto R, Pilato B, Palumbo O, Merla G, Simone G and Tommasi S: MiR-578 and miR-573 as potential players in BRCA-related breast cancer angiogenesis. Oncotarget 6: 471-483, 2015.

50. BaiR, Weng C, Dong H,Li S, Chen G and Xu Z: MicroRNA-409-3p suppresses colorectal cancer invasion and metastasis partly by targeting GAB1 expression. Int J Cancer 137: 2310-2322, 2015.

This work is licensed under a Creative Commons Attribution-NonCommercial-NoDerivatives 4.0 International (CC BY-NC-ND 4.0) License. 